BMJ Open Sport \& Exercise Medicine

\title{
Fifth metatarsal stress fracture in elite male football players: an on-field analysis of plantar loading
}

\author{
Athol Thomson, ${ }^{1,2}$ Richard Akenhead, ${ }^{3}$ Rodney Whiteley, ${ }^{4}$ Pieter D'Hooghe, ${ }^{4}$ \\ Ken Van Alsenoy, ${ }^{4}$ Chris Bleakley ${ }^{5}$
}

To cite: Thomson $A$, Akenhead R, Whiteley R, et al. Fifth metatarsal stress fracture in elite male football players: an on-field analysis of plantar loading. BMJ Open Sport \& Exercise Medicine 2018;4:e000377. doi:10.1136/ bmjsem-2018-000377

Accepted 31 May 2018

\section{Check for updates}

${ }^{1}$ Exercise and Sports Science Department, Aspetar Orthopaedic and Sports Medicine Hospital, Doha, Qatar 2Ulster Sports Academy, University of Ulster, Ulster, UK ${ }^{3}$ Football Association, Burton Upon Trent, UK

${ }^{4}$ Rehabilitation Department, Aspetar Orthopaedic and Sports Medicine Hospital, Doha, Qatar ${ }^{5}$ Department of Physical Therapy, School of Health Sciences, High Point University, High Point, North Carolina, USA

Correspondence to Athol Thomson; Athol. Thomson@Aspetar.com

\section{ABSTRACT}

Objective Evaluate plantar loading during 'on-field' common football movements in players after fifth metatarsal (MT-5) stress fracture and compare with matched healthy players.

Methods Fourteen elite male soccer players participated in the study conducted on a natural grass playing surface using firm ground football boots. Seven players who had suffered a primary stress fracture (MT- 5 group) and seven matched healthy players (controls, CON) performed three common football movements while in-shoe plantar loading data were collected.

Results Large between-group differences exist for maximal vertical force normalised to bodyweight $\left(\mathrm{F}_{\max }\right)$ at the lateral toes $(2-5)$ of the stance leg during a set-piece kick (MT-5: 0.2 \pm 0.06 bodyweight (BW), CON: $0.1 \pm 0.05$ BW, effect size (ES) 1.4) and the curved run where the MT-5 group showed higher $F_{\text {max }}$ with very large effect size at the lateral forefoot of the injured (closest to curve) limb when running a curve to receive a pass (MT5 injured-CON=0.01 BW, ES 1.5). Small between-group differences were evident during straight-line running. However, between-limb analysis of MT- 5 group showed significant unloading of the lateral forefoot region of the involved foot.

Conclusions Elite male football players who have returned to play after MT-5 stress fracture display significantly higher maximum plantar force at the lateral forefoot and lateral toes (2-5) compared with healthy matched control players during two football movements (kick and curved run) with the magnitude of these differences being very large. These findings may have important implications for manipulating regional load during rehabilitation or should a player report lateral forefoot prodromal symptoms.

\section{INTRODUCTION}

Return to sport following fifth metatarsal (MT-5) stress fracture in football (soccer) players can be problematic and protracted. Average absence from football is $3-5$ months when healing and rehabilitation go to plan. ${ }^{1}$ Complications, however, are common with non-union and refracture being among the chief concerns, which makes this injury potentially 'career-ending'.
What are the new findings?

Dynamic plantar loading assessment while performing football-specific on-field movements may be more applicable than static measurement to detect differences in lateral foot loading for football players.

- Understanding the loading profile of common football movements may allow for load manipulation at regional anatomical structures at the lateral aspect of the foot especially in the event that prodromal symptoms are reported by the player.

How might it impact on clinical practice in the near future?

Young midfield players who perform many high-velocity passes or set-piece kicks and report latera foot pain should warrant further investigation.

- These data may help guide earlier 'sports-specific' on-field training after MT-5 surgery by allowing movements that do not impart large loading demands on the lateral aspect of the foot.

Young players, during the preseason period of training, are most affected with the non-dominant (stance leg when kicking) limb more frequently involved in the midfielder playing position. ${ }^{2-4}$ Early surgical intervention with insertion of a large-diameter compression screw is thought to lead to better outcomes for athletes. ${ }^{56}$

Understanding the magnitude, timing and distribution of forces acting at players' feet when performing common football movements is therefore important to minimise the risk of primary injury or refracture after surgical fixation. Greater ground reaction forces and impact loading rates occur when running in football boots compared with training shoes. ${ }^{7}$ In-shoe plantar loading is a proxy of vertical ground reaction force (vGRF) experienced by the player, and while in-shoe systems are known to slightly underestimate peak vGRF compared with force plate measurements, they allow valid and 
reliable collection of multiple steps during 'on-field' testing. ${ }^{8}{ }^{9}$ Additionally, analysis of regional loading at specific anatomical sites of the foot is possible rather than one global measure of vGRF. ${ }^{10-12}$

Football footwear is known to increase plantar loading at specific anatomical areas of the foot during movements like running, cutting and kicking. ${ }^{10-12}$ How these plantar loading parameters are altered following MT-5 stress fracture when compared with healthy matched players during common football movements is unknown.

Therefore, the primary aim of this study is to evaluate plantar loading during 'on-field' common football movements in players after return to sport following MT-5 stress fracture and compare with matched healthy players. A secondary aim was to identify football movements that increase load at the lateral forefoot to guide activity modification should prodromal symptoms be reported to medical personnel.

\section{METHODS}

\section{Participants}

Fourteen elite male soccer players participated in the study. Seven players (professional and/or international players) who had suffered a primary stress fracture of the fifth MT (MT-5 group-age $25 \pm 5$ years, weight $74 \pm 6 \mathrm{~kg}$, height $178 \pm 6 \mathrm{~cm}$ ) and seven matched healthy (no injury) players (control group $-26 \pm 4$ years, $76 \pm 4 \mathrm{~kg}, 179 \pm 5 \mathrm{~cm}$ ) were recruited. All MT-5 injured players underwent surgery at Aspetar Sports Medicine and Orthopaedic Hospital (Doha, Qatar) by the same orthopaedic surgeon (PD) for intramedullary screw fixation \pm bone graft from the pelvis. Postoperative care comprised 3 weeks in a non-weight-bearing cast and 3 weeks in a partial weightbearing boot. Physiotherapy was started after cast removal with combined hydrotherapy and reduced gravity treadmill (Alter-G) in the initial phase. After 6 weeks, all players progressed to full-weight-bearing. For all MT-5 injured players, the affected foot was at their stance or non-dominant limb when kicking with $86 \%(6 / 7)$ of the players reporting prodromal symptoms prior to stress fracture. All stress fractures occurred with insidious onset and were not frank or acute traumatic fractures ${ }^{2}$ (figure 1). Playing positions comprised four midfield players, one wing, one striker and one defender.

Inclusion in the current study occurred only after MT-5 players had returned to play following complete radiographic union of the stress fracture and completion of rehabilitation programme with end-stage field-based return-to-play tests at the rehabilitation department in Aspetar Sports Medicine and Orthopaedic Hospital. MT-5 group players were on average $240 \pm 60$ days after surgery during on-field biomechanical testing.

Healthy matched control participants were injury-free for 6 months prior to the study with no previous history of injury to MT- 5 or anterior cruciate ligaments. Healthy participants were matched for playing position, body mass, height and level of competition.

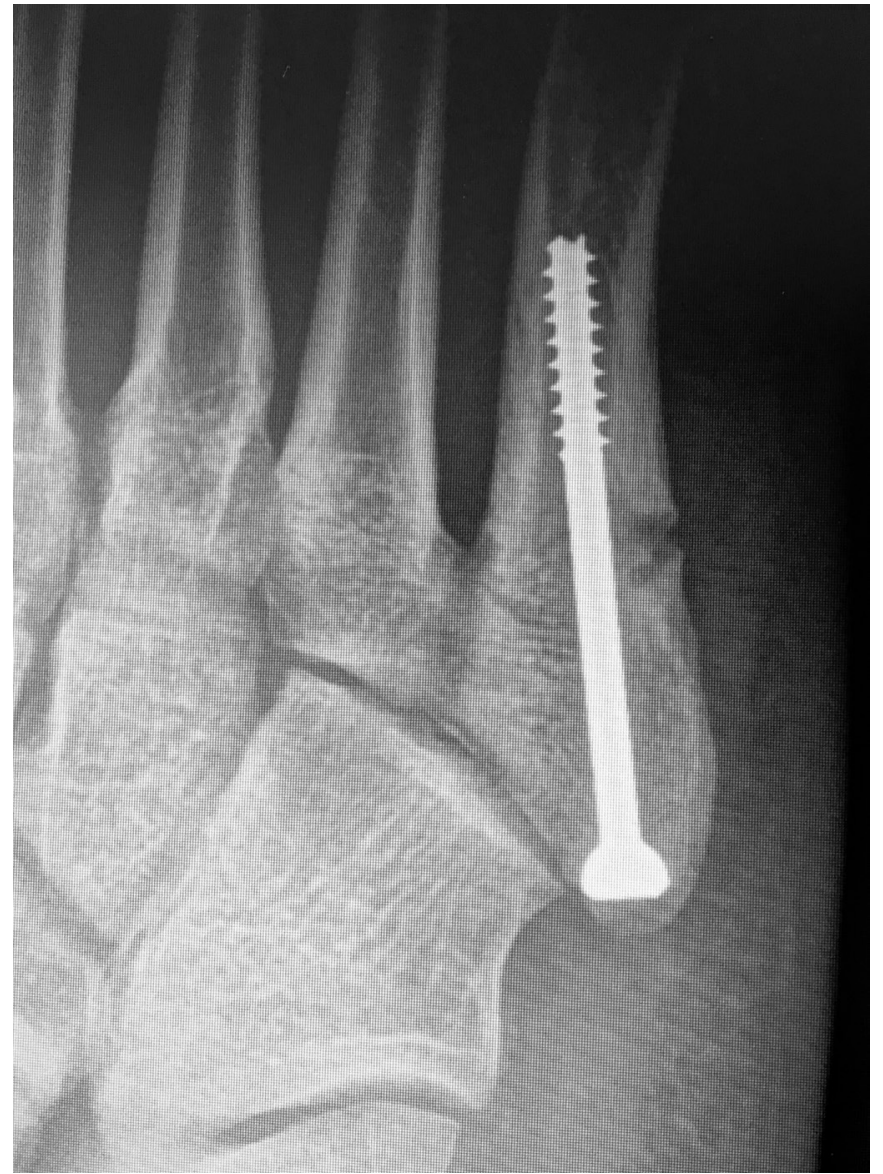

Figure 1 X-ray of one players' fifth metatarsal stress fracture (insidious onset) after surgical fixation with an intramedullary screw. Note location distal to the tuberosity where traumatic avulsion fractures occur.

\section{Testing protocol}

Participants were fitted with appropriate-sized firm ground soccer boots (Nike Tiempo Genio leather II; Nike, Beaverton, Oregon, USA) for field-based biomechanical testing (figure 2). Natural Bermuda grass (Cynodon dactylon) surface over-seeded with rye grass (Lolium perenne) with a predominantly sand rootzone at the Aspire zone (Doha, Qatar) was used for testing. Ground staff maintained the surface to have consistent mechanical properties for the duration of the study. ${ }^{13}$ Surface hardness $(69 \pm 6 \mathrm{~g}$ using FIFA-approved $2.25 \mathrm{~kg}$ Clegg hammer), rotational resistance $(43 \pm 7 \mathrm{Nm}$ using FIFA-approved studded disc apparatus) and temperature (26 $\pm 6{ }^{\circ} \mathrm{C}$ using Kestrel 4400 heat stress tracker, USA) were recorded.

Plantar loading parameters were collected using the Pedar-X in-shoe system (Novel, Munich, Germany). Each insole is $1.9 \mathrm{~mm}$ thick and contains 99 capacitive sensors, which were calibrated prior to testing (Trublu Calibration; Novel). The validity and reproducibility of Pedar-X is excellent for running. ${ }^{9} 14$

Briefly, the Pedar-X insoles relay data sampled at 100 $\mathrm{Hz}$ to a data logger (carried in a custom-made back-pack) and then to a laptop via Bluetooth technology. Insoles 


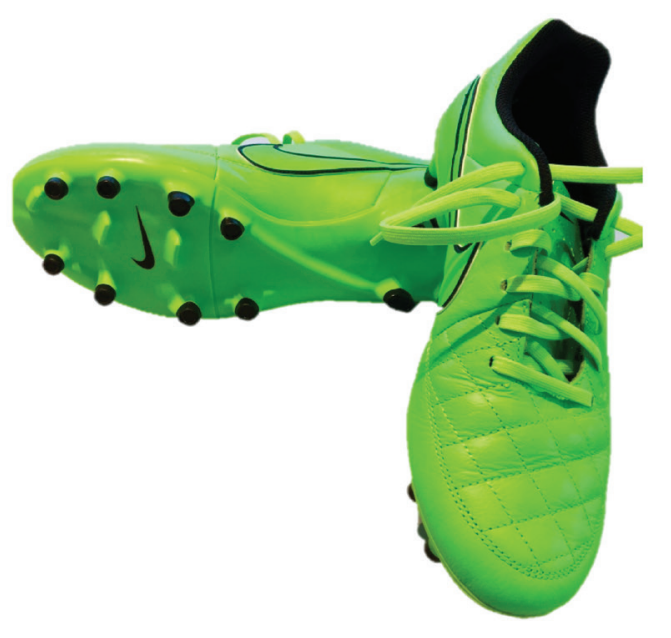

Figure 2 Firm ground football shoe used by all participants (Nike Tiempo Genio II).

are placed bilaterally with no foot orthotics in place so that the Pedar-X insoles were flat. A global positioning system (GPS) with accelerometer (Catapult, Australia) was used to verify running speed of the trials.

Standardised 'warm-up' protocol consisting of progressing running speeds, lower body resistance exercises (bodyweight, BW) and dynamic stretching was conducted by the same physical performance coach (RA). Following this, participants completed three soccer football-specific movement tests (figure 3). For each test, three familiarisation trials were first performed, followed by three trials of each test while kinetic and spatiotemporal data were collected via the Pedar-X and GPS unit as follows:

1. Set-piece kick: Participants were instructed to hit the furthermost top corner of the goal posts from a spot $10 \mathrm{~m}$ adjacent to the corner of the 18 yd box during three trials of curved set-piece kicks at $75 \%$ of maximum effort while data were collected from the stance leg.

2. Curved run with ball interplay: Participants performed three curved runs to mimic running into space onto a pass at around $75 \%$ of maximum effort. Participants dribbled the football to a cone where they passed to a stationary team-mate (RA) who sent out a subsequent pass for the participant to run onto while following the arc of the centre circle (figure 2). Participants were instructed to accelerate into the curved run, after passing the ball, at $75 \%$ of maximum effort.

3. Forward straight-line run: Participants ran $60 \mathrm{~m}$ at a speed of $5.5 \mathrm{~m} \mathrm{~s}^{-1}(19.8 \mathrm{~km} / \mathrm{h})$. Running speed was controlled using audio cues in which the participant should pass each $10 \mathrm{~m}$ distance marker cone as the audio cue (beep) sounds. Speed was checked with the GPS system and any trials outside $\pm 10 \%$ were discarded.

For the straight run, plantar loading data from the stance phase of a minimum of six consecutive footfalls were extracted for both the left and right feet and were averaged for subsequent analysis using Novel evaluation
A

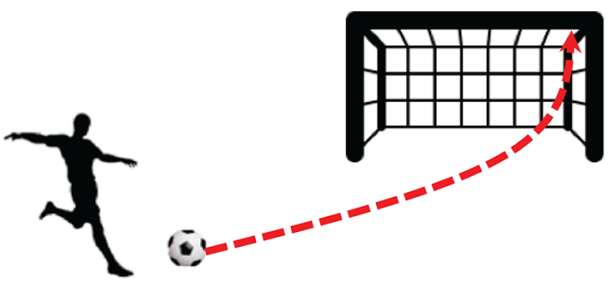

B
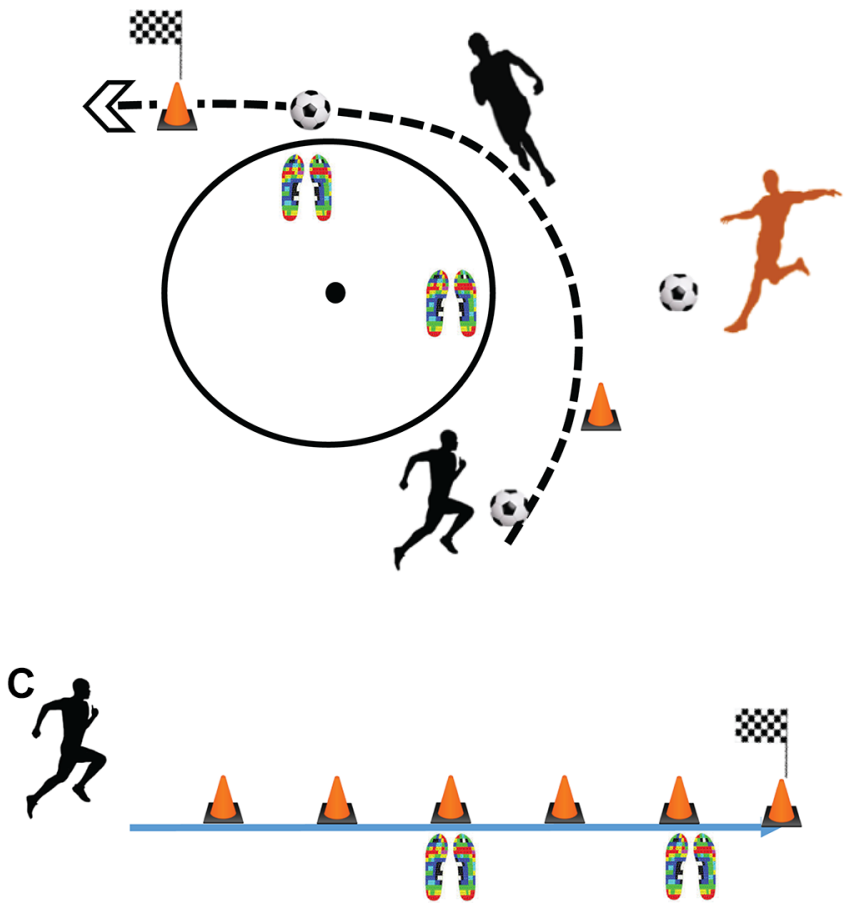

Figure 3 Football-specific movements. (A) Set-piece kick. (B) Curved run with ball interplay. (C) Forward straight-line run at $5.5 \mathrm{~m} \mathrm{~s}^{-1}$. The pressure insole icons denote areas where data collection started and finished during the running trials.

software (Groupmask Evaluation; Novel). For the curved run with ball interplay, the maximum force $\left(\mathrm{F}_{\max }\right)$ of the inside foot (closest to the curve) was averaged over the three trials. For the set-piece kick, the $\mathrm{F}_{\text {max }}$ at the non-dominant stance leg was averaged over the three trials. The $\mathrm{F}_{\text {max }}$ was normalised to each participant's bodyweight to facilitate between-participant comparison and was examined for the whole foot as well as anatomical regional areas ('masks') for each task (figure 4). ${ }^{15}$ $\mathrm{F}_{\text {max }}$ recorded by Pedar-X is a proxy measure of vertical ground reaction force (vGRF) and has been shown to correlate well with a Kistler force platform. ${ }^{8}$ The betweenlimb difference for $\mathrm{F}_{\text {max }}$ was calculated subtracting the value of the MT-5 injured limb from the uninjured limb for each MT-5 participant, and arbitrarily for the healthy participants as right leg subtracted from the left leg. Data for the whole foot and also anatomical masks were analysed with a focus on the lateral foot. The masks examined were 'lateral midfoot', 'lateral forefoot' and 'lateral toes $(2-5)$ '. 

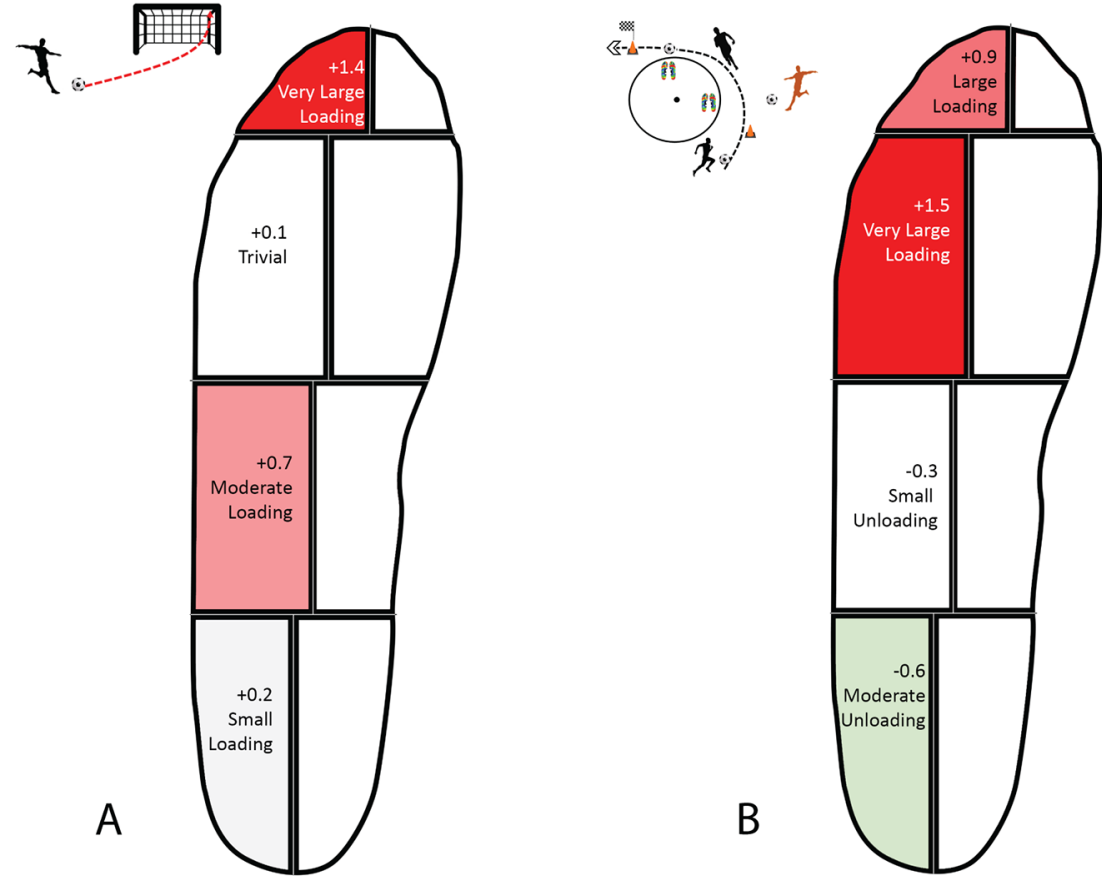

Increase

Fmax

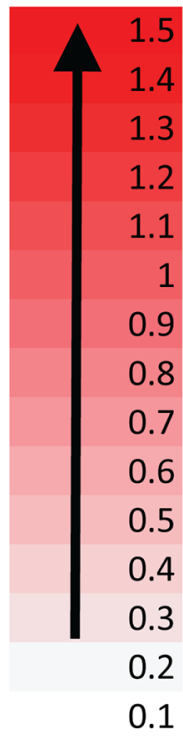

0

$-0.1$
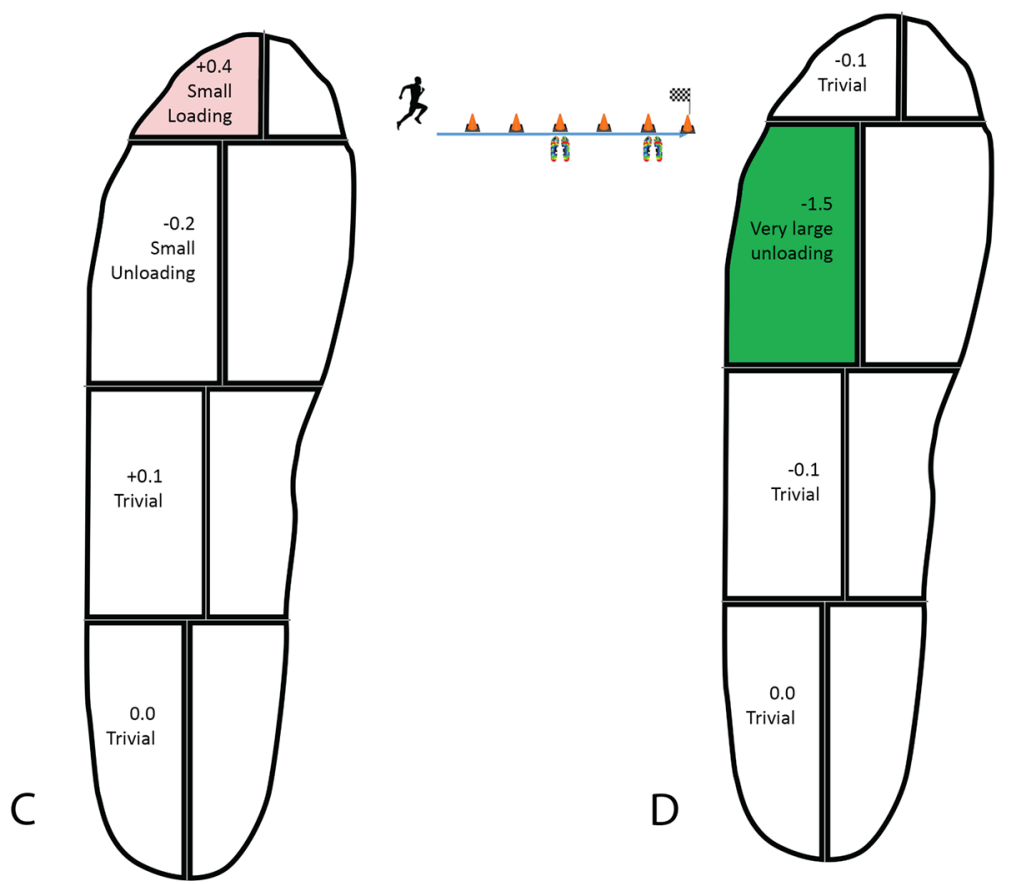

$-0.2$

$-0.3$

$-0.4$

$-0.5$

$-0.6$

$-0.7$

$-0.8$

$-0.9$

$-1$

$-1.1$

$-1.2$

$-1.3$

$-1.4$

Decrease

Fmax

Figure 4 Between-group differences (MT-5 injured group vs control group) for specific anatomical regions of the foot expressed as effect sizes (Cohen's d). (A) Set-piece kick. (B) Curved run with ball interplay. (C) Forward straight-line run at 5.5 $\mathrm{m} \mathrm{s}^{-1}$. (D) Between-limb difference (within the MT-5 injured group) during a forward straight-line run at $5.5 \mathrm{~m} \mathrm{~s}^{-1}$.

\section{Statistical analysis}

Between-limb and between-group differences were examined with an analysis of variance and subsequent post hoc testing with $\mathrm{p}<0.05$ set as indicating statistical significance. Between-group differences were reported using Cohen's d. ${ }^{16}$ The differences were reported as small, medium, large and very large when they reached $0.2,0.5$, 0.8 and 1.2 , respectively. ${ }^{17}$

\section{RESULTS}

Between-group differences at the lateral foot for each movement task are presented in table 1. Differences for each anatomical region of interest are expressed as effect sizes (Cohen's d) in figure 4A-C.

Between-limb differences within the MT-5 group for the forward run task are presented in table 2 and figure 4D. 
Table 1 Maximum force (normalised to BW) for each anatomical region during three movement tasks

\begin{tabular}{lllcc}
\hline Maximum force (normalised BW) & Anatomical region & Control group & Effect size (Cohen's d) & MT-5 group \\
\hline Set-piece kick & Lateral toes 2-5 & $0.11 \pm 0.05$ & $1.4^{*}$ & $0.20 \pm 0.06$ \\
& Lateral forefoot & $0.60 \pm 0.09$ & 0.1 & $0.62 \pm 0.08$ \\
& Lateral midfoot & $0.79 \pm 0.02$ & 0.7 & $0.95 \pm 0.01$ \\
& Total foot & $2.93 \pm 0.31$ & 0.8 & $3.29 \pm 0.50$ \\
Curved run with ball & Lateral toes 2-5 & $0.17 \pm 0.09$ & 0.9 & $0.25 \pm 0.08$ \\
& Lateral forefoot & $0.75 \pm 0.10$ & $1.5^{\star}$ & $0.89 \pm 0.05$ \\
& Lateral midfoot & $0.88 \pm 0.02$ & -0.3 & $0.82 \pm 0.02$ \\
Forward striaght-line run & Total foot & $3.04 \pm 0.32$ & 0.9 & $3.30 \pm 0.19$ \\
& Lateral toes 2-5 & $0.20 \pm 0.01$ & 0.4 & $0.24 \pm 0.09$ \\
& Lateral forefoot & $0.78 \pm 0.01$ & -0.2 & $0.76 \pm 0.02$ \\
& Lateral midfoot & $0.67 \pm 0.01$ & 0.1 & $0.68 \pm 0.02$ \\
& Total foot & $2.96 \pm 0.20$ & 0 & $2.96 \pm 0.30$ \\
\hline
\end{tabular}

Between-group differences (MT-5 group injured limb-control group equivalent limb or average of R and L limbs) expressed as effect size.

Red data bars=increased force for MT-5 group. Green bars=decreased force for MT-5 group.

*Significant difference between groups $(p<0.05)$.

BW, bodyweight.

\section{Set-piece kick}

Significant differences (stance leg of MT-5 group-stance leg of control (CON) group) are reported for each lateral anatomical region.

Lateral midfoot: Increase in $\mathrm{F}_{\max }$ compared with the control group with moderate ES (MT-5 $1.0 \pm 0.01 \mathrm{BW}$, CON 0.8 \pm 0.02 BW, ES 0.7, p>0.05).

Total foot: Overall, for the total foot, the MT-5 group produced higher $\mathrm{F}_{\max }$ than the control group during a set-piece kick (MT-5 3.3 \pm 0.6 BW, CON 2.9 \pm 0.3 BW, ES $0.8, \mathrm{p}>0.05)$.

Lateral toes 2-5: Substantial increase in $\mathrm{F}_{\max }$ (MT-5 $0.2 \pm 0.06 \mathrm{BW}, \mathrm{CON} 0.1 \pm 0.05 \mathrm{BW}$, effect size (ES) 1.4, $\mathrm{p}=0.03$ ) with very large effect size when kicking.

Table 2 Maximum force (normalised to BW) for each anatomical region during a forward straight-line run at at 5.5 $\mathrm{m} \mathrm{s}^{-1}(19.8 \mathrm{~km} / \mathrm{h})$

\begin{tabular}{lllll}
\hline $\begin{array}{l}\text { Maximum } \\
\text { force } \\
\text { (normalised } \\
\text { BW) }\end{array}$ & $\begin{array}{l}\text { Anatomical } \\
\text { region }\end{array}$ & $\begin{array}{l}\text { MT-5 group } \\
\text { uninvolved } \\
\text { limb }\end{array}$ & $\begin{array}{l}\text { Effect size } \\
\text { (Cohen's d) }\end{array}$ & $\begin{array}{l}\text { MT-5 group } \\
\text { injured limb }\end{array}$ \\
\hline $\begin{array}{l}\text { Forward } \\
\text { straight-line } \\
\text { run }\end{array}$ & $\begin{array}{l}\text { Lateral toes } \\
2-5\end{array}$ & $0.24 \pm 0.09$ & -0.1 & $0.23 \pm 0.07$ \\
& $\begin{array}{l}\text { Lateral } \\
\text { forefoot }\end{array}$ & $0.89 \pm 0.01$ & $-1.5^{*}$ & $0.76 \pm 0.01$ \\
& $\begin{array}{l}\text { Lateral } \\
\text { midfoot } \\
\text { Total foot }\end{array}$ & $0.70 \pm 0.17$ & -0.1 & $0.69 \pm 0.19$ \\
& $2.96 \pm 0.30$ & -0.2 & $2.94 \pm 0.19$ \\
\hline
\end{tabular}

In inter-limb difference within the MT-5 group (injured-uninvolved limb). Green data bars and negative effect size show magnitude of unloading at the injured limb.

*Significant difference between groups $(p<0.05)$.

BW, bodyweight.

\section{Curved run with ball interplay}

Significant differences (MT-5 injured limb-control group inside limb when running towards curve) are reported for each lateral anatomical area.

Lateral forefoot: MT-5 group showed higher $\mathrm{F}_{\max }$ with very large effect size at the lateral forefoot of the inside (closest to curve) limb when running a curve to receive a ball (MT-5 injured-CON=0.01 BW, ES 1.5, $\mathrm{p}=0.004$ ).

Lateral toes 2-5: Higher $\mathrm{F}_{\max }$ with large effect size compared with the control group (MT-5 injured$\mathrm{CON}=0.005$ BW, ES 0.9, p>0.05).

Total foot: Overall for the total foot, the MT-5 group had higher $\mathrm{F}_{\max }$ with large effect size than the control group (MT-5 injured-CON=0.02 BW, ES 0.9, p>0.05).

\section{Forward straight-line run at $5.5 \mathrm{~m} \mathrm{~s}^{-1}$}

Between-group analysis (MT-5 injured limb-average of both control players' limbs)

Lateral toes 2-5: $\mathrm{F}_{\max }(0.04 \mathrm{BW}$, ES 0.4, p>0.05) increased with small effect sizes compared with the control group.

MT-5 group between-limb analysis (injured limb-uninjured limb) Lateral forefoot: An unloading strategy was apparent for the previously injured MT-5 limb in comparison with the healthy limb of the MT-5 group players with a substantial decrease in $\mathrm{F}_{\max }$ (MT-5 injured-healthy limb $=0.13$ BW) at the lateral forefoot with the magnitude of effect being very large $(\mathrm{ES}=-1.5, \mathrm{p}=0.03$ ) (figure $4 \mathrm{D}$ and table 2).

Total foot: Overall, for the total foot, a decrease in $\mathrm{F}_{\max }$ with small effect size was noted when comparing the MT-5 injured limb with the healthy limb for a forward run at $5.5 \mathrm{~m} \mathrm{~s}^{-1}$ (MT-5 injured-healthy limb=0.06 BW, ES $-0.2, \mathrm{p}>0.05)$. 


\section{DISCUSSION}

To the authors' knowledge, this is the first study to examine 'on-field' game relevant movements of elite football players (using football boots) who have returned to play after surgical fixation of a fifth metatarsal stress fracture and compared the kinetic data with healthy matched control players.

Football-specific movements (set-piece kick and curved run with ball interplay) showed much larger betweengroup differences at the lateral aspect of the foot than a straight-line running task in this cohort of male football players. Recent prospective research $(\mathrm{n}=335)$ by Matsuda et $a t^{4}$ implied static plantar pressure measurements are not effective for identifying those players who will go on to develop a MT-5 stress fracture. Alongside current data presented here, we further advocate the need for assessment during on-field game relevant movements rather than static posture or even straight-line running alone.

Lateral maximum force was highest for the MT-5 group players at the stance leg during the set-piece kick or the inside foot when accelerating into a curved run (figure 3A and B). However, straight-line running at $5.5 \mathrm{~m} \mathrm{~s}^{-1}$ (19.8 $\mathrm{km} / \mathrm{h}$ ) showed very little plantar load at the lateral foot other than the lateral toes (2-5) (figure 3C). Previous research in healthy football players indicated increased plantar loading at the medial (not lateral) forefoot when cutting, running at moderate speeds and sprinting. ${ }^{12} 18$ This information may allow players to stay involved at training with certain movement strategy modifications should they report lateral foot pain.

Between-limb comparison within the MT-5 injured group showed an 'unloading' strategy at the lateral forefoot (figure 3D) when running straight. The contrast in lateral loading when compared with the other movement tasks may represent an inability of previously injured MT-5 players to 'stress-shield' or unload the area once the task becomes more challenging. This finding is similar to pressure plate laboratory barefoot walking research conducted on 10 professional football players after they had returned to sport following MT-5 stress fracture. ${ }^{19}$

\section{High index of suspicion with prodromal signs}

Prodromal symptoms (such as vague lateral foot pain) might provide an important window of opportunity to intervene and manipulate an individual's loading variables following intense blocks of training. ${ }^{2}{ }^{20}$ Eighty-six per cent $(6 / 7)$ of participants in the MT-5 group reported prodromal symptoms prior to full stress fracture. It appears that these symptoms are frequently encountered: Ekstrand and Van Dijk ${ }^{2}$ reported $45 \%$ of players who sustain a MT-5 fracture reported prodromal symptoms at the lateral foot and Popovic et $a l^{21}$ noted all 17 players had prodromal symptoms prior to stress fracture in a surgically managed cohort. Provided medical teams have a 'player wellness' monitoring system in place, early intervention may be possible. From the current findings, it is suggested that accelerating into curved runs towards the injured foot or performing set-piece kicks that curl towards a target such as high ball velocity crosses, corner kicks and set-piece penalty kicks may substantially increase lateral loading and should be monitored until symptoms have resolved (table 1). Previous research suggests crossover cutting may also be viewed with caution due to increases in lateral foot pressure. ${ }^{22}$

Maximum plantar force alone is likely not a sufficient metric to be used as an indicator for when stress fracture will ensue due to the multifactorial nature of lower limb injuries, and it is suggested that the volume of kick type $^{23}$ as well as running and direction change demands ${ }^{24}$ should be individualised to player position when considering return to sport programming. These data may provide further insight into pathogenesis of MT-5 stress fracture when combined with the current data and other factors (eg, sleep quality, training load, vitamin D status, match congestion, anatomical variation including local vascularity, and player age). ${ }^{1-325}$

\section{Toe-flexor strength and management of ground reaction force}

Decreased toe-grip strength measured with a digital dynamometer in a large prospective cohort of male football players $(n=273)$ was found to be a prospective risk factor in players who went on to develop MT-5 stress fracture. ${ }^{3}$ The lateral toes $2-5$ anatomical region showed much larger magnitudes of $\mathrm{F}_{\text {max }}$ in the MT- 5 group for all three movements tested here, peaking with the set-piece kick (figure 3A). This suggests the external vGRF is greater at the lateral toes in injured players, which might be a result of kicking technique (more foot inversion and staying lateral through roll-over progression to toe-off) at the stance leg during kicking. External GRFs must be absorbed and managed by internal forces via the lateral plantar fascia, peroneus brevis/tertius muscle-tendon unit attachments and bending moments at the MT-5 bone itself. Decreased toe-flexor strength may incur higher bending moment forces on the MT-5 bone due to inability to manage the external GRF that generate large torsion, tension and axial loads especially when the foot is inverted prior to contact such as during full effort set-piece kicking. ${ }^{26} 27$

\section{Football boots}

All players wore identical soccer boots (figure 2). While this helped control for the effect different footwear might have on plantar loading, it also means that footwear was not tailored to the individual's foot anatomy or preference. Given the large magnitude of lateral loading with the football-specific tasks, it is suggested that stud plate outsole width at the midfoot and forefoot must be wide enough to prevent lateral 'overhang' of the MT-5 bone in an attempt to offer some form of protection from the playing surface. Queen $e t a l^{22}$ suggested the addition of midsole cushioning, increased number of studs and decreased stud length reduced forefoot pressure during two football-specific tasks (side cut and cross cut) when using turf shoes instead of football boots. Additionally, clinical experience of the authors 
suggest footwear companies should perhaps work to incorporate forefoot cushioning into the outsole of football boots as many international and professional players remove the football shoe insole (sock liner) completely in attempt to improve 'feel' for the ball by wearing very tight shoes.

Further studies are required, with larger sample size, to assess if early intervention and manipulation of loading variables following prodromal symptoms does indeed reduce progression to stress fracture or if these findings extend to other populations (women, adolescents, older players and different playing levels).

An obvious limitation of this study in that 'in-shoe' systems measure vertical force and hence we miss the medial-lateral and anterior-posterior components of GRF. Cross-sectional design of this study should also be considered when interpreting the results as we cannot discern whether the higher lateral loads seen in the MT-5 group were the cause of stress fracture or a consequence of the injury.

However, even after completing a graduated rehabilitation programme and having returned to previous level of competitive football, the MT- 5 injured players examined here displayed large differences in plantar loading at the lateral foot. These data should implore practitioners to exercise a high index of suspicion should prodromal symptoms occur in a player with history of previous MT-5 stress fracture and manipulate football movements accordingly.

\section{CONCLUSIONS}

Elite male football players who have returned to play after MT-5 stress fracture display significantly higher maximum plantar force at the lateral forefoot and lateral toes (2-5) compared with healthy matched control players during two football movements (kick and curved run) with the magnitude of these differences being very large. These findings may have important implications for manipulating regional load during rehabilitation or should a player report lateral forefoot prodromal symptoms.

\section{Twitter @ATholThomson}

Acknowledgements The authors would like to thank the players for agreeing to take part in this study along with Professor Mathew Wilson, Haitham Ahmed and Wayne Holmes for technical and logistical support.

Contributors AT and RA conceived the study. All authors collected data and wrote the manuscript. RW and KVA performed the statistical analysis. PD shared surgical expertise. All authors approved the final manuscript.

Funding The authors have not declared a specific grant for this research from any funding agency in the public, commercial or not-for-profit sectors.

Competing interests None declared.

Patient consent Obtained.

Ethics approval This study was approved by the ethics committee of the AntiDoping Lab Qatar institutional review board (IRB no. F2016000194).

Provenance and peer review Not commissioned; externally peer reviewed.

Data sharing statement A full dataset of all plantar loading parameters can be provided on request.
Open access This is an Open Access article distributed in accordance with the Creative Commons Attribution Non Commercial (CC BY-NC 4.0) license, which permits others to distribute, remix, adapt, build upon this work non-commercially, and license their derivative works on different terms, provided the original work is properly cited and the use is non-commercial. See: http://creativecommons.org/ licenses/by-nc/4.0/

(c) Article author(s) (or their employer(s) unless otherwise stated in the text of the article) 2018. All rights reserved. No commercial use is permitted unless otherwise expressly granted.

\section{REFERENCES}

1. Ekstrand J, Torstveit MK. Stress fractures in elite male football players. Scand J Med Sci Sports $2012 ; ; 22: 341-6$. Jun 1.

2. Ekstrand J, van Dijk CN. Fifth metatarsal fractures among male professional footballers: a potential career-ending disease. $\mathrm{Br} J$ Sports Med 2013;47:754-8.

3. Fujitaka K, Taniguchi A, Isomoto S, et al. Pathogenesis of fifth metatarsal fractures in college soccer players. Orthop J Sports Med 2015;3:232596711560365.

4. Matsuda S, Fukubayashi T, Hirose N. Characteristics of the foot static alignment and the plantar pressure associated with fifth metatarsal stress fracture history in male soccer players: a casecontrol study. Sports Med Open $2017 ; ; 3: 27$. Dec 1.

5. Kerkhoffs GM, Versteegh VE, Sierevelt IN, et al. Treatment of proximal metatarsal $\mathrm{V}$ fractures in athletes and non-athletes. $\mathrm{Br} \mathrm{J}$ Sports Med 2012;46:644-. Jan 1.

6. Porter DA, Duncan M, Meyer SJ. Fifth metatarsal Jones fracture fixation with a $4.5-\mathrm{mm}$ cannulated stainless steel screw in the competitive and recreational athlete: a clinical and radiographic evaluation. Am J Sports Med 2005 ;;33:726-33. May.

7. Smith N, Dyson R, Janaway L. Ground reaction force measures when running in soccer boots and soccer training shoes on a natural turf surface. Sports Engineering 2004;7:159-67.

8. Barnett S, Cunningham JL, West S. A comparison of vertical force and temporal parameters produced by an in-shoe pressure measuring system and a force platform. Clin Biomech 2000;15:781-5.

9. Stöggl T, Martiner A. Validation of Moticon's OpenGo sensor insoles during gait, jumps, balance and cross-country skiing specific imitation movements. J Sports Sci 2017;35:196-206.

10. Bentley JA, Ramanathan AK, Arnold GP, et al. Harmful cleats of football boots: a biomechanical evaluation. Foot Ankle Surg 2011;17317:140-4.

11. Ford KR, Manson NA, Evans BJ, et al. Comparison of in-shoe foot loading patterns on natural grass and synthetic turf. J Sci Med Sport $2006 ;$;9:433-40. Dec 1.

12. Eils E, Streyl M, Linnenbecker S, et al. Characteristic plantar pressure distribution patterns during soccer-specific movements. Am J Sports Med 2004;32:140-5.

13. Caple M, James I, Bartlett M. Mechanical behaviour of natural turf sports pitches across a season. Sports Engineering $2012 ; ; 15: 129-41$. Sep 1.

14. Kernozek TW, Zimmer KA. Reliability and running speed effects of in-shoe loading measurements during slow treadmill running. Foot Ankle Int $2000 ;$;21:749-52. Sep.

15. Girard O, Racinais S, Kelly L, et al. Repeated sprinting on natural grass impairs vertical stiffness but does not alter plantar loading in soccer players. Eur J Appl Physiol 2011;111:2547-55.

16. Cohen J. A power primer. Psychol Bull 1992;112:155-

17. Sawilowsky SS. New effect size rules of thumb. J Mod Appl Stat Meth 2009;8:597-9.

18. Wong PL, Chamari K, Mao DW, et al. Higher plantar pressure on the medial side in four soccer-related movements. Br J Sports Med 2007;41:93-100.

19. Hetsroni I, Nyska M, Ben-Sira D, et al. Analysis of foot structure in athletes sustaining proximal fifth metatarsal stress fracture. Foot Ankle Int 2010;31:203-11.

20. Eirale C. Hamstring injuries are increasing in men's professional football: every cloud has a silver lining? Br J Sports Med 2018:bjsp orts-2017-098778. Jan 23.

21. Popovic N, Jalali A, Georis P, et al. Proximal fifth metatarsal diaphyseal stress fracture in football players. Foot and Ankle Surgery 2005;11:135-41.

22. Queen RM, Charnock BL, Garrett WE, et al. A comparison of cleat types during two football-specific tasks on FieldTurf. Br J Sports Med 2008;42:278-84.

23. Whiteley R, Farooq A, Johnson A. Development of a databased interval kicking program for preparation and rehabilitation 
purposes in professional football. Science and Medicine in Football 2017;1:107-16.

24. Mohr M, Krustrup P, Bangsbo J. Match performance of highstandard soccer players with special reference to development of fatigue. J Sports Sci 2003;21:519-28.

25. Warden SJ, Davis IS, Fredericson M. Management and prevention of bone stress injuries in long-distance runners. J Orthop Sports Phys Ther 2014;44:749-65.
26. Gu YD, Ren XJ, Li JS, et al. Computer simulation of stress distribution in the metatarsals at different inversion landing angles using the finite element method. Int Orthop 2010;34:669-76.

27. Vertullo CJ, Glisson RR, Nunley JA. Torsional strains in the proximal fifth metatarsal: implications for Jones and stress fracture management. Foot Ankle Int 2004;25:650-6. 